\title{
A Cultural Algorithm for the Representation of Mitochondrial Population
}

\author{
Athanasios Alexiou and Panayiotis Vlamos \\ Department of Informatics, Ionian University, Plateia Tsirigoti 7, 49100 Corfu, Greece \\ Correspondence should be addressed to Athanasios Alexiou, alexiou@ionio.gr \\ Received 31 May 2012; Accepted 14 August 2012 \\ Academic Editor: Catalina Cocianu
}

Copyright ( $) 2012$ A. Alexiou and P. Vlamos. This is an open access article distributed under the Creative Commons Attribution License, which permits unrestricted use, distribution, and reproduction in any medium, provided the original work is properly cited.

We propose a novel Cultural Algorithm for the representation of mitochondrial population in mammalian cells as an autonomous culture. While mitochondrial dysfunctions are highly associated with neurodegenerative diseases and related disorders, an alternative theoretical framework is described for the representation of mitochondrial dynamics. A new perspective of bioinspired algorithm is produced, combining the particle-based Brownian dynamics simulation and the combinatorial representation of mitochondrial population in the lattice, involving the optimization problem of ATP production in mammalian cells.

\section{Introduction}

Considering the latest researches, disruptions in the regulation of mitochondrial dynamics, low-energy production, increased reactive oxygen species, and mtDNA damage are relevant to human diseases, mainly in neurogenerative diseases and cancer. Recent discoveries have highlighted that neurons are reliant particularly on the dynamic properties of mitochondria. In addition, mitochondria are actively recruited to subcellular sites, such as the axonal and dendritic processes of neurons. Defects in mitochondrial dynamics are associated with neurodegenerative disease. For example, Charcot-Marie-Tooth type 2A, a peripheral neuropathy, and dominant optic atrophy, an inherited optic neuropathy, result from a primary deficiency of mitochondrial fusion. Moreover, several major neurodegenerative diseases including Parkinson's, Alzheimer's, and Huntington's diseases involve disruption of mitochondrial dynamics.

On the other hand, cultural algorithms are a class of population concepts, principles, mechanisms, and optimization techniques that work on a principle inspired by nature: evolution of species [1]. These algorithms are very useful tools in a large number of applications in optimization, control, signal processing, or machine learning [2].

Lately, researchers attempted to model the cultural evolution process from both a microevolutionary perspective in terms of the transmission of behaviors or traits between individuals in a population and a macroevolutionary perspective in terms of the formation of generalized beliefs based upon individual experiences. These generalized beliefs can serve to constrain the behaviors of individuals within the associated population [3].

According to Reynolds, Cultural Algorithms are a class of computational models of cultural evolution that support such a dual inheritance perspective. This approach provides a framework in which to describe all of the current models of cultural evolution from a computational point of view since any of the single inheritance systems can be produced as a special case [3].

Cultural Algorithms, are based on the supposition that one can get better learning rates for an evolutive genetic algorithm [4] adding to it one more element of evolutive pressure-called Belief Space, a mechanism of cultural pressure. Therefore, a system of double inheritance, both genetic and cultural, could better respond to a large number of problems, while cultural evolution enables societies to evolve or adapt to their environments at rates that exceed that of biological evolution based upon genetic inheritance alone $[5,6]$.

In this paper we investigate the application of Cultural Algorithms on the representation of mitochondrial population in mammalian cells, a biological system with high 
complexity, different capabilities and operations, and great importance for the human health.

\section{The Mitochondrial Population and Dynamics}

The number of mitochondria in a cell is regulated to match the cell's requirements for ATP, while fusions and fissions play a functional role in maintenance of proper inner membrane electrical potential. Mitochondria provide most of the ATP for cellular reactions. ATP production in mitochondria is coupled to an electron transport system in which the passage of electrons down the various electron carriers is associated with the transport of protons from the matrix into the intermembrane space. The majority of these protons reenter the mitochondrial matrix by the ATP syntheses, thereby generating ATP.

Mitochondria are involved in numerous metabolic and cellular processes [7]. Besides the citric acid cycle and the oxidative phosphorylation, these processes also include the urea cycle and the oxidation of fatty acids. Other reactions carried out and orchestrated are the biosynthesis of heme, several amino acids, and vitamin cofactors, as well as the formation and export of iron-sulphur clusters [8]. Beyond these metabolic functions, mitochondria are also involved in the programmed cell death [9] and in case of their dysfunction in ageing [10] and several diseases [11].

2.1. Mitochondrial Structure. Found in most eukaryotic cells, mitochondria are subcellular organelles that play a central role in energy metabolism. The key feature of the mitochondrion is the presence of two membranes that encapsulate a protein-rich central matrix. Specifically, this organelle is compartmentalized by two membranes into four compartments. A smooth outer membrane surrounds and isolates the organelle from the cytosol while the inner membrane with several invaginations, called cristae, divides them further into the mitochondrial intermembrane space and the matrix. The organization of the inner membrane was dissected in recent studies using improved electron microscopic and tomographic techniques $[12,13]$. The inner membrane contains the protein complexes and redox cofactors involved in electron transfer and ATP synthesis.

Besides the outer membrane, an inner boundary membrane is connected by several tubular junctions to the cristae, creating a distinction between the intermembrane and the intercristal space. This basic concept of the inner membrane is structurally dynamic with respect to cristae connection to each other or with the inner membrane and can be considerably varied among different organisms' tissues or physiological conditions [12]. The mitochondrial matrix contains a highly concentrated mixture of enzymes involved in all aspects of metabolism, in addition to the mitochondrial genome which, in mammalian mitochondria, encodes 39 genes involved in mitochondrial function. Families of mitochondrial carriers, of which approximately 50 have been identified in the human genome, are present to enable exchange between the intermembrane space and the matrix [14].
The fact that many central processes in eukaryotic cells are functionally linked to this double membrane shielded organelle requires an interface between the mitochondrial compartment and the cytoplasm. To achieve this intracellular exchange the two membranes include a variety of transport and receptor proteins [15-17] as well as a specific subset of translocases involved in the import and assembly of mitochondrial proteins [18].

2.2. Mitochondrial Processes: Fusion, Fission, Motility, and Mitophagy. The number of mitochondria in a cell is regulated to match the cell's requirements for ATP, while fusions and fissions play a functional role in maintenance of proper inner membrane electrical potential. Without the mitochondrial dynamics, the mitochondrial population consists of autonomous organelles that have impaired function [19]. In a wild type cell, high rates of fusion and fission are independent events, which constantly change the identity of individual mitochondria, as well as the motility and the mitophagy.

An individual mitochondrion is not an autonomous organelle. The hundreds of mitochondria within a typical cell undergo continual cycles of fusion and fission. Because mitochondria have an outer lipid membrane as well as an inner one, each fusion event requires the coordinated fusion of the membranes [20,21]. Fusion is likely to protect function by providing a chance for mitochondria to mix their contents, thus enabling protein complementation, mtDNA repair, and equal distribution of metabolites, helping the isolation of damaged-mitochondrial segments and promoting their autophagy $[22,23]$. In contrast, fission acts in order to facilitate equal segregation of mitochondria into daughter cells during cell division and to enhance distribution of mitochondria along cytoskeletal tracks [24].

Intuitively, it is easy to imagine how mitochondrial fusion and fission can change the morphologic characteristics of mitochondria [20-22]. Fusion results in fewer and longer mitochondria, whereas fission results in more and shorter mitochondria. Indeed, genetic studies indicate that cells with mutations in the genes required for mitochondrial fusion have fragmented mitochondria instead of the tubular mitochondrial network observed in normal cells [24]. Similarly, cells with mutations in genes required for mitochondrial fission have excessively elongated and interconnected mitochondria because of unopposed fusion. Peroxisomal shape is also controlled by fission; the role of fusion is less clear [20].

Additional studies have also shown that mitochondrial fission precedes apoptosis [25]. Defects in mitochondrial fusion cause neurodegenerative disease [23, 24]. CharcotMarie-Tooth disease type $2 \mathrm{~A}$, an autosomal dominant neuropathy of long peripheral nerves, is caused by mutations in MFN2. Moreover, dominant optic atrophy, the most commonly inherited optic neuropathy, is caused by mutations in OPA1. This apparent sensitivity of neurons to defects in mitochondrial dynamics probably depends on the special requirements of neurons for mitochondrial function [20]. An ultrastructural hallmark of the synapse is the presence of abundant mitochondria, which maintains calcium homeostasis and levels of ATP production that, 
in turn, are critical to nerve transmission. Neurons have extraordinarily long cellular processes, and tight control of mitochondrial dynamics is probably necessary for distributing active mitochondria to dendrites and axon terminals. Given the important roles of mitochondrial dynamics in human physiologic processes, it would not be surprising to find additional diseases caused by mutations in genes that control mitochondrial fusion and fission [20].

Another aspect of mitochondrial dynamics beyond fusion and fission is the motility of mitochondria [21]. This aspect is critically important in highly polarized cells, such as neurons [26], which require mitochondria at sites distant from the cell body, but can also be crucial to cellular function in smaller cells [27]. Defects in both fusion and fission have been shown to decrease mitochondrial movement. In neurons lacking mitochondrial fusion, both increased mitochondrial diameter due to swelling and aggregations of mitochondria seem to block efficient entry into neurites, resulting in a dearth of mitochondria in axons and dendrites [28]. These defects result in improperly developed neurons or gradual neurodegeneration.

Autophagy is a mechanism whereby eukaryotic cells degrade their own cytoplasm and organelles [29]. Autophagy functions as a homeostatic nonlethal stress response mechanism for recycling proteins to protect cells from low supplies of nutrients and as a cell death mechanism. This degradation of organelles and long-lived proteins is carried out by the lysosomal system; thus, a hallmark of autophagy is accumulation of autophagic vacuoles of lysosomal origin [23]. Autophagy has been seen in developmental and pathological conditions. Mitophagy denotes the degradation of mitochondria through autophagy. Although the existence of mitophagy has been known for some time, it has been unclear whether mitochondria are randomly or selectively targeted for mitophagy [21].

\section{An Alternative Biological Culture}

3.1. Mitochondrial Civilization Associated with Neurogenerative Diseases. From a geometrical point of view, a number of (un)correlated factors can affect the mitochondrial shape. This illustrates a more complex problem, while morphological changes in mitochondrial structure are associated with biological dysfunctionalities and electrophysiology problems [30]. These effects are directly or indirectly correlated with human neurodegenerative diseases. While fusions and fissions contribute to the wide variety of mitochondrial morphologies, a discrete mitochondrion at one point in time will be changed at a later time by the addition of new mitochondrial material through fusion or by the removal of material through division. It is a logical consequence of high probability that after a certain period of successful events (fusions and fissions) the inner structure will totally lose its initial characteristics in a nonreversible way, restricting the inner space and reducing the corresponding area and energy [30]. It is obvious that any failure in inner membrane mitochondrial fissions can easily generate unstable electric potential, effecting functionality and reduce voltage gradient. Fusion and fission seems to be required to maintain mitochondrial function, as independent and different mechanisms. Fusion is likely to protect function by providing a chance for mitochondria to mix their contents, thus enabling protein complementation, mtDNA repair, and equal distribution of metabolites, helping the isolation of damaged mitochondrial segments and promoting their autophagy. In contrast, fission acts in order to facilitate equal segregation of mitochondria into daughter cells during cell division and to enhance distribution of mitochondria along cytoskeletal tracks. The failure in this biological machinery may also promote apoptosis.

Even though, these four processes are independent, it is clear that any interactions will be critically important in neurons. For example, defects in both fusion and fission have been shown to decrease mitochondrial movement. The large tangle of highly interconnected mitochondria in fission-deficient cells prevents efficient movement, especially into small pathways such as neuronal processes. While mitophagy denotes the degradation of mitochondria through autophagy, recent findings indicate that mitophagy can selectively degrade defective mitochondria.

Especially in the case of Alzheimer's Disease, scientists used brain tissue from cases with a diagnosis of $\mathrm{AD}[31,32]$, as well as control cases with no clinical or pathological history of neurological disease, applying cytological in situ hybridization, immunocytochemistry, and morphometry [33] techniques, showing that the area of intact mitochondria is significantly decreased in $\mathrm{AD}$. While $\mathrm{AD}$ can be genetically classified as familiar or sporadic, researchers proposed that the case of sporadic $\mathrm{AD}$ is not caused by the accumulation of amyloid- $\beta(\mathrm{A} \beta)$, but instead is a consequence of a decline in mitochondrial function with age $[34,35]$. Additionally, the overexpression of $A \beta$ causes an alteration in the mitochondrial fission and fusion proteins resulting in mitochondrial dysfunction, mitochondrial fragmentation, increase in reactive oxygen species (ROS) and ATP production, and reduced mitochondrial membrane potential [36].

3.2. A New Cultural Algorithm. From the philosopher Aristotle to the anthropologist Geertz [37], any civilization consists of citizens, where besides the variation of their genetic characteristics, they participate to the social evolution through their behaviour and several others mechanisms, rules, principles, or even more principles.

Additionally researchers [38] introduce the term Artificial Culture where, a new knowledge domain tries to connect the models found in complex adaptive systems to the models found in the domain of culture. According to this terminology Artificial Culture is a population of individual agents, with its own sense, with its own cognition and performance, interacting in a social ambient with others agents in a physical environment of artifacts and others objects [38].

According to Holland [39], a complex system usually has the following characteristics, which obviously in the case of mitochondrial populations are adapted in most of the cases.

(i) Relationships in complex system are nonlinear.

(ii) Complex systems contain feedback loops. 
$t \leftarrow 1$

Generate Civilization: $N$ individuals are distributed in the parametric space, assuming that correspond to a local martingale Initialize Population $\mathrm{P}(t)$

Initialize Belief Space BS $(t)$

While $($ ATP Production $=$ Acceptant $)$ and $(t<$ constant value $)$ do \{

Evaluate $\mathrm{P}(t)$

Evolve $((\mathrm{P}(t)$, Merging $(\mathrm{P}(t)$, Combine $(\mathrm{P}(t))))$, Influence $(\mathrm{BLF}(t)))$

Vote $(\mathrm{BS}(t), \operatorname{Accept} t(\mathrm{P}(t)))$

Evaluate $(\mathrm{P}(t)$, Adjust $(\mathrm{BS}(t)))$

Update $(\mathrm{BS}(t)$, Accept $(\mathrm{P}(t)))$

$t \leftarrow t+1$

Select $\mathrm{P}(t)$ from $\mathrm{P}(t-1)$

\}

End

Algorithm 1: Cultural Algorithm.

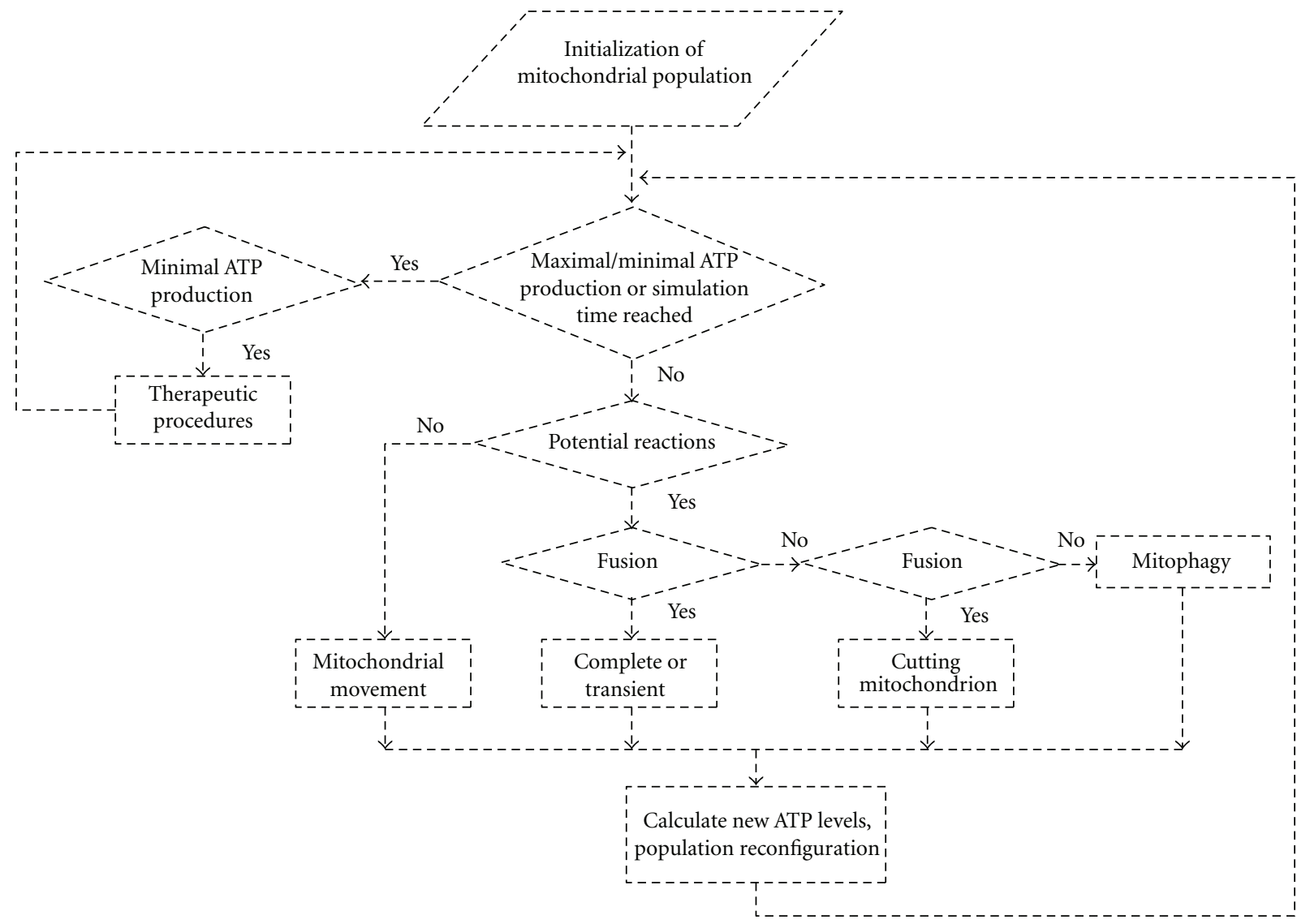

FIgURE 1: The Cultural Algorithm for the representation of mitochondrial population.

(iii) Complex systems are open.

(iv) Complex systems have memory (even though mitochondria seem to obey the memoryless phenomenon [30]).

(v) Complex system may produce emergent phenomena.
The proposed Cultural Algorithm for the representation of the mitochondrial population is an extended version of Reynolds [3], adapted in mitochondrial terminology (operations) where $\mathrm{P}(t)$ represents the population and $\mathrm{BS}(t)$ the Belief Space at time $t$.

The algorithm starts with the generation of the civilization, where the initialization of both the Population and 


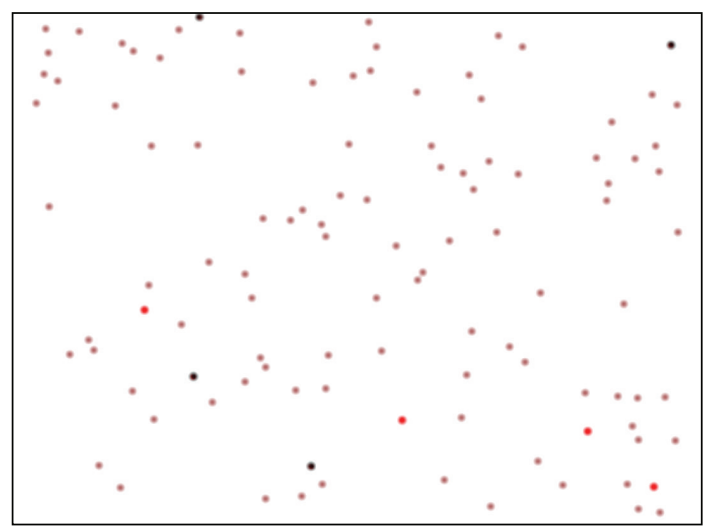

(a)

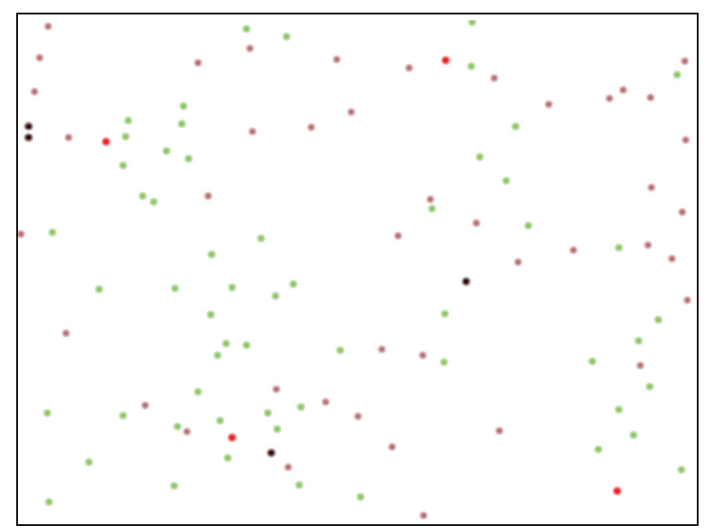

(b)

FIgURE 2: (a) Initialization of population. (b) Simulation results after $N$ Steps.

the Belief Space, occurs. Due to the biological identities of mitochondria in mammalian cells, and their stochastic distribution in the lattice space, we assumed that they produce a local martingale limiting their ATP production within the normal measurement limits. The proposed algorithm enters the evolution loop until the ATP production is decreased in a level likely to result in a neurological disorder. At the beginning of each generation, individuals in the Population Space are first evaluated and then interact to each other through the operation factors "Merging" and "Combine" (representing in a way the mitochondrial dynamics and the communication paths between them in order to exchange their contents) resulting a new selection of individuals through the Influence function.

Additionally, the two feedback functions Accept and Influence, give the opportunity to the population component and the Belief Space to interact with each other in the way that human culture evolve [40-42].

The pseudocode of the proposed bioinspired algorithm is given in Algorithm 1.

By the mechanism of mitochondrial dynamics these organelles can undergo exchange their contents for desired morphology and topology. The above algorithm is a more sophisticated control of mitochondrial proper functioning, setting as population belief a total optimum in the cell space.

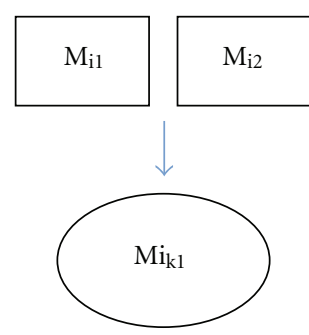

(a)
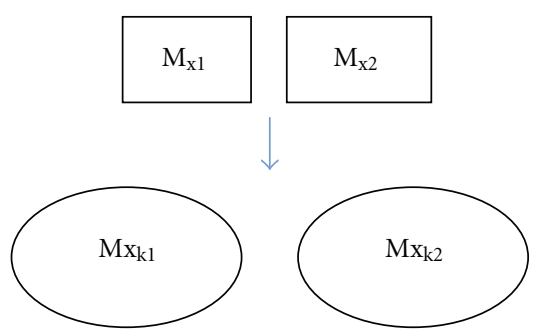

(b)

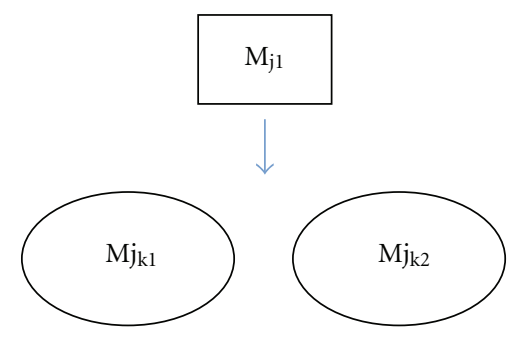

(c)

Figure 3: (a) Complete fusion. (b) Transient fusion. (c) Fission.

Empirically, fusion-deficient mitochondria display loss of directed movement, instead hovering in a manner reminiscent of Brownian motion [43]. It is well known that particle-based Brownian dynamics simulations offer the opportunity to not only simulate diffusion of particles but also the reactions between them. Particle-based simulations naturally incorporate the concepts of space, crowding, and stochasticity $[44,45]$. Those methods treat proteins or other reactants explicitly, and the time-evolution of particle positions is sampled at discrete time intervals by Brownian dynamics simulations $[46,47]$. The basic motion of a particle undergoing diffusion can be described by Einstein's diffusion equation [48] as follows:

$$
\frac{\partial}{\partial t} P\left(r, t \mid r_{0}, t_{0}\right)=D \nabla^{2} P\left(r, t \mid r_{0}, t_{0}\right)
$$

where $P\left(r, t \mid r_{0}, t_{0}\right)$ is the probability that the particle will be at position $r$ at time $t$ given the particle was initially at position $r_{0}$ at time $t_{0}$. The rate of diffusion is given by the parameter $D$. When the diffusive motions of multiple particles are simulated, in a traditional Brownian dynamics algorithm the distribution of particle displacements $P(r, t)$ is sampled for each particle every time step [49].

Algorithm 1 is a a novel theoretical approach in order to represent mitochondrial population of mammalian cells as an independent culture. We assigned a random walk in 
these subcellular compartments of a neural cell as follows: viruses to destroyed mitochondria, infected individuals to mitochondria with decreased ATP production, and uninfected individuals to healthy mitochondria.

A more informative representation of the above pseudocode can be seen in Figure 1, where the main feature involves the optimization level of ATP production. In this Cultural Algorithm the stochastic evolution function is performed until the solution has reached a total optimum, the maximal or minimal ATP level, responsible for several human diseases.

We tested our algorithmic representations in a multiagent simulation environment called MASON, which is a single-process discrete-event simulation core and visualization library for biological systems [50].

The population is represented by the colour dots [50], brown for the healthy mitochondria, green for the mitochondria with decreased functionality, and the red for the destroyed mitochondria. There are also some cases of healthy mitochondria, which are represented with black dots with red cross, where destroyed mitochondria can be disinfected through the operation of fusion (Figure 2).

It is obvious that the formulation of mitochondrial population as a biological culture, gives us the potentiality of visualization of the two main observed classes of fusion events in mammalian cells. The complete fusion and the transient fusion events [51], wherein two mitochondria came into close apposition, exchanged soluble intermembrane space and matrix proteins, and reseparated, preserving the original morphology.

In this phase, we are more focused in the three main mitochondrial operations: the complete fusion, the transient fusion of two mitochondria, and the fission of one mitochondrion (Figure 3).

It is obvious that further simulation testing of these dependent operations will give us the opportunity of modelling efficiently the mitochondrial dysfunctions over age and figure more accurate the potential safe boundaries of healthy mitochondrial population against the early diagnosis of neurogenerative diseases. Genes, proteins interactions, and mtDNA quality, shall be taken into consideration in future work as the main factors of the proposed representation affecting the mitochondrial population and its functionality.

\section{Conclusions and Future Work}

Cultural Algorithms will definitely offer a powerful evolutionary algorithmic tool for many biological diseases at both the levels of diagnosis and treatment. In the case of mitochondrial populations and their association with several neurogenerative disorders, this computation technique will lead us to a more formalistic representation of experimental results concerning subcellular measurements and mitochondrial population. In this paper we presented a new combined version of Cultural Algorithms, using the basic notions of evolutionary algorithms on mitochondrial dynamics.

Future research includes more computational tests and a detailed analysis of the performance on real biological data and case studies, concerning mammalian cells.

\section{References}

[1] K. J. Batenburg, "An evolutionary algorithm for discrete tomography," Discrete Applied Mathematics, vol. 151, no. 1-3, pp. 36-54, 2005.

[2] K. J. Batenburg and W. J. Palenstijn, "A new exam timetabling algorithm," in Proceedings of the 15th Belgium-Netherlands Artificial Intelligence Conference (BNAIC'03), T. Heskes, P. Lucas, L. Vuurpijl, and W. Wiegerinck, Eds., pp. 19-26, 2003.

[3] R. G. Reynolds, "An introduction to cultural algorithms," in Proceedings of the 3rd Annual Conference on Evolutionary Programming, pp. 131-139, 1994.

[4] D. E. Goldberg, Genetic Algorithms, Addison-Wesley Longman, USA, 1998.

[5] R. G. Reynolds, "An introdution to cultural algorithms," Cultural Algorithms Repository, 1998.

[6] R. G. Reynolds, E. Zannoni, and R. M. Posner, "Learning to understand software using cultural algorithms," Cultural Algorithms Repository, 1998.

[7] A. S. Reichert and W. Neupert, "Mitochondriomics or what makes us breathe," Trends in Genetics, vol. 20, no. 11, pp. 555562, 2004.

[8] R. Lill and G. Kispal, "Maturation of cellular Fe-S proteins: an essential function of mitochondria," Trends in Biochemical Sciences, vol. 25, no. 8, pp. 352-356, 2000.

[9] G. Vandecasteele, G. Szabadkai, and R. Rizzuto, "Mitochondrial calcium homeostasis: mechanisms and molecules," IUBMB Life, vol. 52, no. 3-5, pp. 213-219, 2002.

[10] A. Trifunovic, "Mitochondrial DNA and ageing," Biochimica et Biophysica Acta, vol. 1757, no. 5-6, pp. 611-617, 2006.

[11] D. C. Wallace, "Mitochondrial diseases in man and mouse," Science, vol. 283, no. 5407, pp. 1482-1488, 1999.

[12] C. A. Mannella, "The relevance of mitochondrial membrane topology to mitochondrial function," Biochimica et Biophysica Acta, vol. 1762, no. 2, pp. 140-147, 2006.

[13] G. Perkins, C. Renken, M. E. Martone, S. J. Young, M. Ellisman, and T. Frey, "Electron tomography of neuronal mitochondria: three-dimensional structure and organization of cristae and membrane contacts," Journal of Structural Biology, vol. 119, no. 3, pp. 260-272, 1997.

[14] E. R. S. Kunji, "The role and structure of mitochondrial carriers," FEBS Letters, vol. 564, no. 3, pp. 239-244, 2004.

[15] R. Lill and G. Kispal, "Mitochondrial ABC transporters," Research in Microbiology, vol. 152, no. 3-4, pp. 331-340, 2001.

[16] B. O'Rourke, "Mitochondrial ion channels," Annual Review of Physiology, vol. 69, pp. 19-49, 2007.

[17] L. Palmieri, F. M. Lasorsa, A. Vozza et al., "Identification and functions of new transporters in yeast mitochondria," Biochimica et Biophysica Acta, vol. 1459, no. 2-3, pp. 363-369, 2000.

[18] W. Neupert and J. M. Herrmann, "Translocation of proteins into mitochondria," Annual Review of Biochemistry, vol. 76, pp. 723-749, 2007.

[19] D. C. Chan, "Mitochondrial fusion and fission in mammals," Annual Review of Cell and Developmental Biology, vol. 22, pp. 79-99, 2006.

[20] D. C. Chan, "Mitochondrial dynamics in disease," New England Journal of Medicine, vol. 356, no. 17, pp. 1707-1709, 2007.

[21] H. Chen and D. C. Chan, "Mitochondrial dynamics-fusion, fission, movement, and mitophagy - in neurodegenerative diseases," Human Molecular Genetics, vol. 18, no. 2, pp. R169176, 2009. 
[22] G. Twig, A. Elorza, A. J. A. Molina et al., "Fission and selective fusion govern mitochondrial segregation and elimination by autophagy," EMBO Journal, vol. 27, no. 2, pp. 433-446, 2008.

[23] A. Alexiou, J. Rekkas, and P. Vlamos, "Modeling the mitochondrial dysfunction in neurogenerative diseases due to high $\mathrm{H}+$ concentration," Bioinformation, vol. 6, no. 5, pp. 173-175, 2011.

[24] L. J. Martin, "Mitochondrial and cell death mechanisms in neurodegenerative diseases," Pharmaceuticals, vol. 3, pp. 839$915,2010$.

[25] R. J. Youle and M. Karbowski, "Mitochondrial fission in apoptosis," Nature Reviews Molecular Cell Biology, vol. 6, no. 8, pp. 657-663, 2005.

[26] P. J. Hollenbeck and W. M. Saxton, "The axonal transport of mitochondria," Journal of Cell Science, vol. 118, no. 23, pp. 5411-5419, 2005.

[27] S. Campello, R. A. Lacalle, M. Bettella, S. Mañes, L. Scorrano, and A. Viola, "Orchestration of lymphocyte chemotaxis by mitochondrial dynamics," Journal of Experimental Medicine, vol. 203, no. 13, pp. 2879-2886, 2006.

[28] H. Chen, J. M. McCaffery, and D. C. Chan, "Mitochondrial fusion protects against neurodegeneration in the cerebellum," Cell, vol. 130, no. 3, pp. 548-562, 2007.

[29] D. J. Klionsky and S. D. Emr, "Autophagy as a regulated pathway of cellular degradation," Science, vol. 290, no. 5497, pp. 1717-1721, 2000.

[30] A. T. Alexiou, P. M. Vlamos, and K. G. Volikas, "A theoretical artificial approach on reducing mitochondrial abnormalities in Alzheimer's disease," in Proceedings of the 10th International Conference on Information Technology and Applications in Biomedicine: Emerging Technologies for Patient Specific Healthcare (ITAB'10), Corfu, Greece, November 2010.

[31] Z. S. Khachaturian, "Diagnosis of Alzheimer's disease," Archives of Neurology, vol. 42, no. 11, pp. 1097-1105, 1985.

[32] S. S. Mirra, A. Heyman, D. McKeel et al., "The Consortium to establish a registry for Alzheimer's disease (CERAD). Part II. Standardization of the neuropathologic assessment of Alzheimer's disease," Neurology, vol. 41, no. 4, pp. 479-486, 1991.

[33] K. Hirai, G. Aliev, A. Nunomura et al., "Mitochondrial abnormalities in Alzheimer's disease," Journal of Neuroscience, vol. 21, no. 9, pp. 3017-3023, 2001.

[34] R. H. Swerdlow and S. M. Khan, "A "mitochondrial cascade hypothesis" for sporadic Alzheimer's disease," Medical Hypotheses, vol. 63, no. 1, pp. 8-20, 2004.

[35] R. H. Swerdlow and S. M. Khan, "The Alzheimer's disease mitochondrial cascade hypothesis: an update," Experimental Neurology, vol. 218, no. 2, pp. 308-315, 2009.

[36] X. Wang, B. Su, H. Fujioka, and X. Zhu, "Dynamin-like protein 1 reduction underlies mitochondrial morphology and distribution abnormalities in fibroblasts from sporadic Alzheimer's disease patients," American Journal of Pathology, vol. 173, no. 2, pp. 470-482, 2008.

[37] C. Geert, A Interpretação Das Culturas, Editora Guanabara, Rio de Janeiro, Brazil, 1989.

[38] N. Gessler, Artificial Culture-Experiments in Synthetic Anthropology, 1999.

[39] J. Holland, Adaptation in Natural and Artificial Systems, MIT Press, Cambridge, Mass, USA, 1992.

[40] J. Barkow, L. Cosmides, and J. Tooby, The Adapted Mind: Evolutionary Psychology and the Generation of Culture, Oxford University Press, USA, 1992.
[41] A. Johnson and T. Earle, The Evolution of Human Societies: from Foraging Group to Agrarian State, Stanford Univ Press, 2000.

[42] P. Richerson and R. Boyd, Not by Genes Alone: How Culture Transformed Human Evolution, University of Chicago Press, 2005.

[43] H. Chen, S. A. Detmer, A. J. Ewald, E. E. Griffin, S. E. Fraser, and D. C. Chan, "Mitofusins Mfn1 and Mfn2 coordinately regulate mitochondrial fusion and are essential for embryonic development," Journal of Cell Biology, vol. 160, no. 2, pp. 189200, 2003.

[44] M. Dobrzyński, J. V. Rodríguez, J. A. Kaandorp, and J. G. Blom, "Computational methods for diffusion-influenced biochemical reactions," Bioinformatics, vol. 23, no. 15, pp. 1969-1977, 2007.

[45] M. Długosz and J. Trylska, "Diffusion in crowded biological environments: applications of Brownian dynamics," BMC Biophysics, vol. 4, no. 1, Article no. 3, 2011.

[46] D. L. Ermak and J. A. McCammon, "Brownian dynamics with hydrodynamic interactions," The Journal of Chemical Physics, vol. 69, no. 4, pp. 1352-1360, 1978.

[47] S. H. Northrup and H. P. Erickson, "Kinetics of proteinprotein association explained by Brownian dynamics computer simulation," Proceedings of the National Academy of Sciences of the United States of America, vol. 89, no. 8, pp. 33383342, 1992.

[48] H. Kim and K. J. Shin, "Exact solution of the reversible diffusion-influenced reaction for an isolated pair in three dimensions," Physical Review Letters, vol. 82, no. 7, pp. 15781581, 1999.

[49] Z. Frazier and F. Alber, "A computational approach to increase time scales in Brownian dynamics-based reaction-diffusion modeling," Journal of Computational Biology, vol. 19, no. 6, pp. 606-618, 2012.

[50] S. Luke, C. Cioffi-Revilla, L. Panait, K. Sullivan, and G. Balan, "MASON: a multiagent simulation environment," Simulation, vol. 81, no. 7, pp. 517-527, 2005.

[51] X. Liu, D. Weaver, O. Shirihai, and G. Hajnóczky, "Mitochondrial kiss-and-run: interplay between mitochondrial motility and fusion-fission dynamics," EMBO Journal, vol. 28, no. 20, pp. 3074-3089, 2009. 

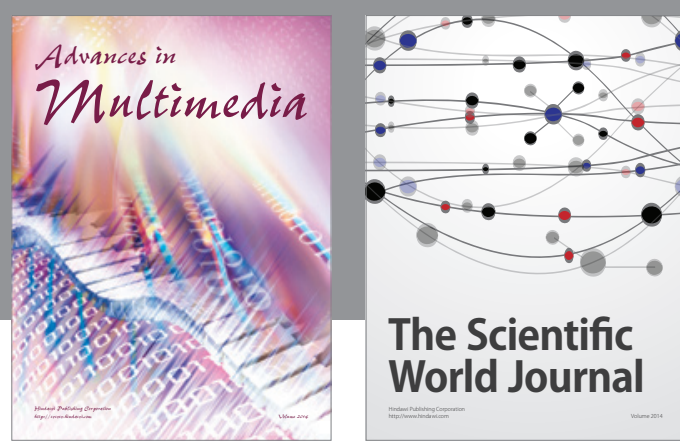

The Scientific World Journal
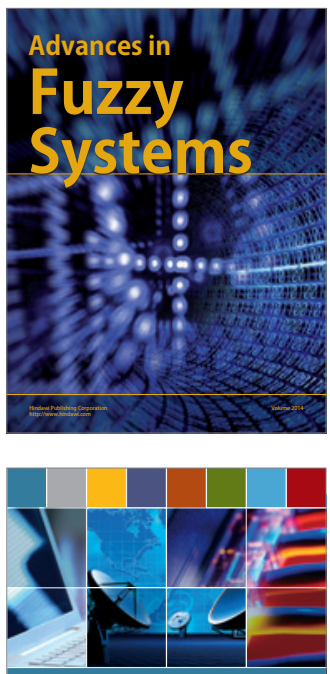

Computer Networks and Communications
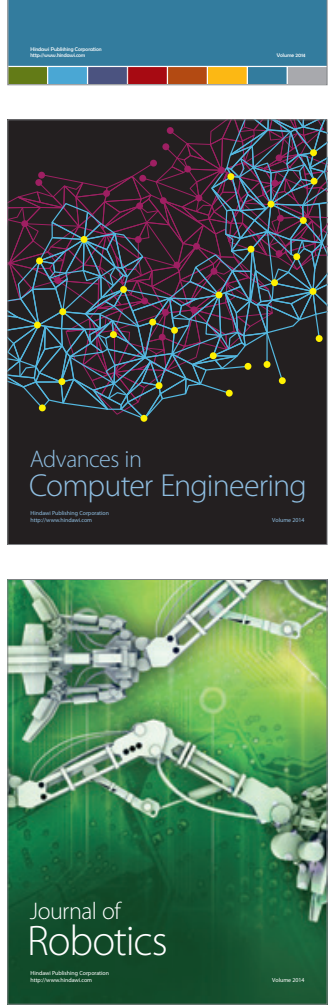
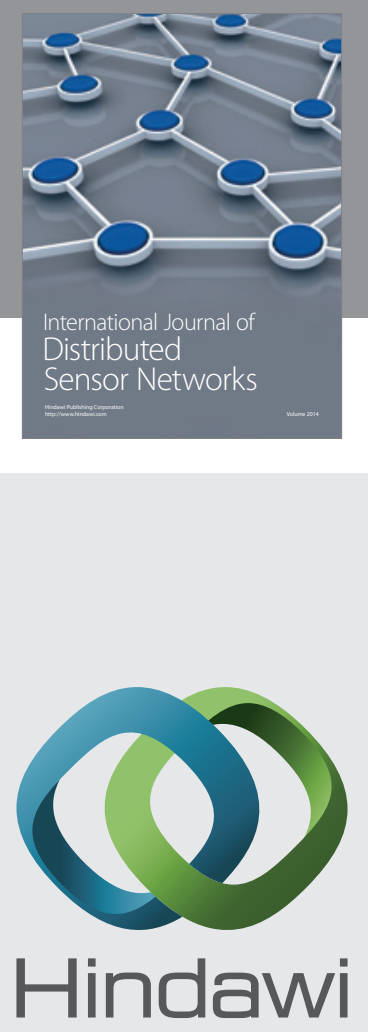

Submit your manuscripts at

http://www.hindawi.com

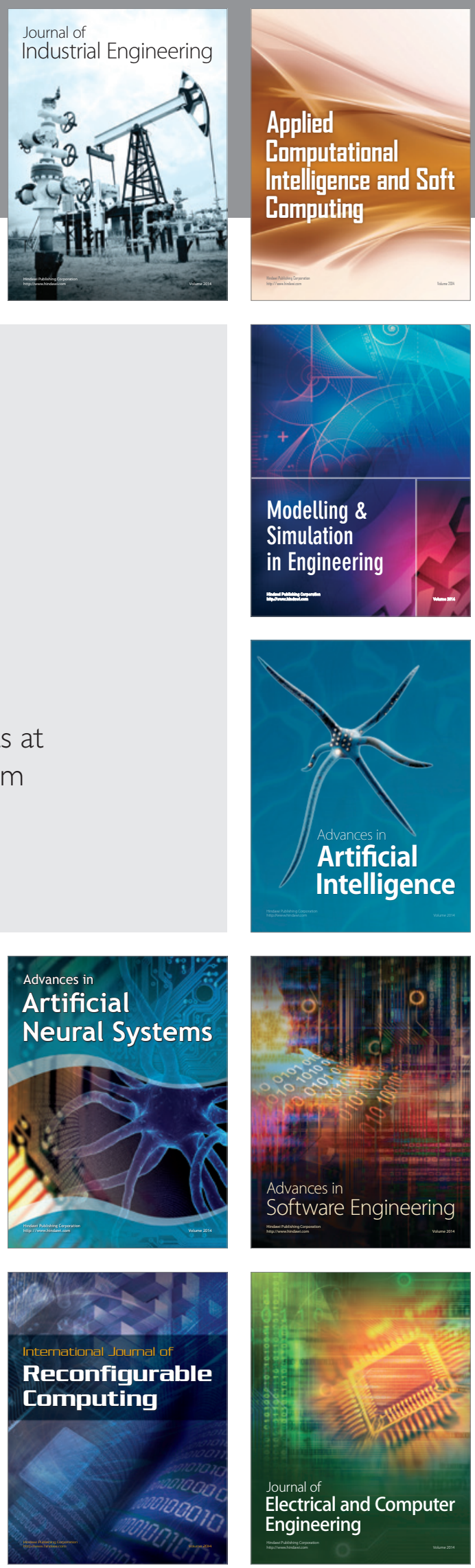